\title{
LES ÉTATS-MAJORS GÉNÉRAUX FRANÇAIS ET LA POLOGNE EN 1938-19391
}

\author{
Simon Catros \\ Université Paris-Sorbonne
}

\section{ABSTRACT \\ THE FRENCH GENERAL STAFFS AND POLAND, 1938-1939}

The weakness and uselessness of the French offensive in Saarland to assist Poland is a sensitive point in the history of the French-Polish relations. This offensive has long been analysed as a demonstration of tactical and operational misconceptions of the French high command in the 1930s. We propose here a new analysis of this offensive on the basis of a study of the relationships between the French general staffs and Poland in the years 1938-1939 and their consequences in strategic conceptions. This study focuses on the role the rapid developments of the strategic balance of power and the diplomatic situation in Europe played during the eighteen months preceding the Second World War. As a result, it is possible to understand the decisive role of the disappearance of the Czechoslovak military factor and the reappraisal of the Soviet military factor in the evolution of the role of Poland in the future conflict as assigned by the French general staffs, and especially by general Gamelin, Chief of Staff for the National Defence.

Key words: Poland, France, French-Polish relations, USSR, Second World War, general staffs, international relations, strategy, General Gamelin.

Mots-clés: Pologne, France, relations franco-polonaises, URSS, Deuxième Guerre mondiale, étatsmajors généraux, relations internationales, stratégie, général Gamelin.

1 Cet article correspond en partie à la transcription d'une communication produite en l'Université Jagellonne dans le cadre du séminaire d'histoire franco-polonaise co-organisé par cette Université et par l'Institut français de Cracovie. Je remercie très chaleureusement ces deux institutions pour leur invitation et leur accueil. 


\section{INTRODUCTION}

La mémoire nationale polonaise est demeurée marquée par la faiblesse de la réaction militaire française en face de l'agression allemande de septembre 1939, couramment perçue comme un abandon. Pour sa part, l'historiographie a longtemps été sévère avec l'attitude militaire de la France envers la Pologne en septembre $1939^{2}$. On observe cependant une inflexion récente vers une meilleure compréhension des considérations tactiques et opérationnelles ayant guidé le haut commandement français ${ }^{3}$. Ces travaux récents mettent également en avant les délais nécessaires à la pleine mobilisation des grandes unités françaises et les conditions politiques - pressions de l'allié britannique contre toute offensive d'ampleur dans les premiers temps du conflit. Cependant, il nous semble utile d'examiner un autre champ de questionnement afin de mieux appréhender les origines et les modalités de l'offensive dans la Sarre. Nous souhaitons ici examiner les relations militaires franco-polonaises durant les mois qui précédèrent la conflagration de septembre 1939 en les croisant avec les considérations stratégiques et opérationnelles encadrant la préparation à la guerre au sein des états-majors généraux (EMG) français.

En effet, dans la mesure où les plans d'opérations militaires étaient du seul ressort du haut commandement ${ }^{4}$, il semble nécessaire d'interroger les relations de ce même haut commandement avec les autorités polonaises, ainsi que sa perception de la place de la Pologne dans la stratégie générale de guerre, pour comprendre la genèse de la réaction militaire française en septembre 1939. Cet article tentera donc de définir la place de la Pologne dans les conceptions stratégiques et opérationnelles des EMG français de mars 1938 à août 1939. Pour conduire cette étude, nous proposerons un nouveau regard sur des archives déjà exploitées, que nous complèterons avec l'analyse d'archives plus récemment mises au jour - fonds d'archives privées du général Gamelin, fonds d'archives militaires rapatriées de Moscou et journal personnel de Paul de Villelume pour la période 1938-1939.

Alors que l'Anschluss rendait de plus en plus évidente la menace allemande sur l'ordre européen, les relations militaires entre Paris et Varsovie entrèrent, paradoxalement, dans une phase profondément perturbée et tendue. Du point de vue des EMG

2 Par exemple : J.-L. Crémieux-Brilhac, Les Français de l'An 40, tome I, « La guerre, oui ou non ? », Paris, Gallimard, «La Suite des temps », 1990, p. 129-131; P. Le Goyet (colonel), Le mystère Gamelin, Paris, Presses de la Cité, 1975, p. 229-234 ; A. Merglen (général), Les forces allemandes sur le front de l'Ouest en septembre 1939, thèse rédigée sous la direction de Georges Dupeux et soutenue en l'Université de Bordeaux, 1969, $161 \mathrm{p}$.

3 M.S. Alexander, The Republic in danger. General Maurice Gamelin and the politics of French defence, 1933-1940, Cambridge, Cambridge UP, 1992, p. 316-322 ; B. Chaix (général), En mai 1940, fallait-il entrer en Belgique? Décisions stratégiques et plans opérationnels de la campagne de France, Paris, Economica, « Campagnes et stratégies. Les grandes batailles », 2000, p. 88-91 ; E.R. May, Strange Victory. Hitler's conquest of France, New York, Hill and Wang, 2000, p. 277-278.

${ }_{4}$ P. de Villelume (général), Journal d'une défaite, 23 août 1939-16 juin 1940, Paris, Fayard, «Les grandes études contemporaines », 1976, p. 214 ; H. Dutailly (colonel), Les problèmes de l'armée de terre française (1935-1939), Paris, Imprimerie Nationale, 1980, p. 28. 
français, une question essentielle était celle du rôle militaire de la Pologne en cas de conflit européen. Les différentes hypothèses à ce sujet étaient fonction des positions politiques et diplomatiques de Varsovie et des capacités militaires prêtées à la Pologne, mais aussi à d'autres puissances d'Europe centrale et orientale, en particulier la Tchécoslovaquie et l'URSS.

Il est nécessaire, à ce stade, de préciser que les forces armées françaises étaient encadrées en 1938-1939 par quatre états-majors généraux : état-major de l'Armée (EMA, forces armées terrestres), état-major-général de la Marine (EMGM, forces navales), état-major de l'Armée de l'Air (EMAA, forces aériennes) et, depuis le 12 mai 1938, état-major des Colonies (EMC, troupes coloniales) ${ }^{5}$. Il sera ici surtout question de l'EMA, qui considéra tout au long des années vingt et trente la Pologne avec un intérêt majeur. Par ailleurs, le chef d'EMG de l'Armée, le général Gamelin, était également chef d'état-major général de la Défense Nationale depuis le début de l'année $1938^{6}$. À ce dernier titre, le général Gamelin était en charge de la coordination stratégique interarmées et de la conduite des conversations militaires avec les puissances étrangères ${ }^{7}$.

Au début de l'année 1938, les relations diplomatiques et militaires franco-polonaises étaient marquées par le legs de deux décennies au cours desquelles alternèrent effusions et refroidissements ${ }^{8}$. Les milieux parisiens avaient été profondément troublés par la déclaration de non-agression germano-polonaise de janvier 1934, couramment soupçonnée de dissimuler une convention militaire secrète ${ }^{9}$. De plus, l'EMA regrettait l'hostilité foncière du cabinet polonais envers la Tchécoslovaquie, qui prenait la forme de campagnes de presse ou de bouderies diplomatiques ${ }^{10}$. Pourtant, à partir

5 SHD, Marine (ci-après : SHD-M), 1BB 2/217, « Nomination d'un chef d'état-major général des Colonies ", 12 mai 1938 ; Service historique de la Défense, Armée de Terre (ci-après : SHD-AT), 2N 223, « Extrait du Journal Officiel du 19 Mai 1938 (Page 5631)»; SHD-AT, 2N 246, « décret du 12 Mai 1938 - Nomination d'un chef d'état-major général des colonies ».

6 SHD-AT, 2N 2, décret portant attributions, en temps de paix, du chef d'état-major général de la Défense Nationale et décret portant nomination du général Gamelin chef d'état-major général de la Défense Nationale, l'un et l'autre datés du 21 janvier 1938.

7 C. Carré (général), Histoire du ministère de la Défense, Panazol, Lavauzelle, 2001, p. 296 ; H. Dutailly (colonel), op. cit., p. 33-34 ; SHD-AT, 2N 224, lettre du général Gamelin aux chefs d'EMG de la Marine et de l'Air, 2 février 1938 ; SHD-AT, 2N 225, procès-verbaux des réunions des chefs d'EMG.

8 Pour le détail des relations diplomatiques et militaires franco-polonaises durant l'Entre-deuxguerres, voir : I. Davion, Mon voisin, cet ennemi, la politique de sécurité française face aux relations polono-tchécoslovaques entre 1919 et 1939, Bruxelles/Berne/Berlin, PIE Peter Lang, « Enjeux internationaux », 2009, 472 p.; F. Dessberg, Le triangle impossible, les relations franco-soviétiques et le facteur polonais dans les questions de sécurité en Europe (1924-1935), Bruxelles, PIE Peter Lang, « Enjeux internationaux », 2009, 440 p. ; N. Jordan, The Popular Front and Central Europe. The dilemmas of French impotence, 1918-1940, Cambridge, Cambridge UP, XV-348 p. ; H. Rollet, La Pologne au $X X^{e}$ siècle, Paris, A. Pedone, 1985, 603 p.

9 L. Noë1, Polonia Restituta, la Pologne entre deux mondes, Paris, Publications de la Sorbonne/ Institut d'Études Slaves, 1984, p. 101 ; SHD-AT, 7N 3015, « Plan de renseignement pour 1934-1935 », section Orient du $2^{\mathrm{e}}$ bureau de l'EMA, probablement rédigé en septembre 1934.

10 SHD-AT, 7N 2522, « Compte-rendu des renseignements fournis par le SR [...] », 2 $2^{\mathrm{e}}$ bureau de l'EMA, 20 novembre et 9 décembre 1937 ; SHD-AT, 7N 3012, « Rapport 146/S du 6 Décembre 1936 du Colonel Attaché Militaire à Varsovie - Analyse », 2e bureau de 1'EMA, sans date ; SHD-AT, 7N 3024, 
de 1935, l'EMA entreprit de redynamiser les relations avec l'armée polonaise. Cette activité se situait dans la continuité de la position de l'EMA de 1925 à 1933, lequel avait toujours affirmé la nécessité d'une entente franco-polonaise, la Pologne représentant à ses yeux le seul contrepoids militaire disponible et efficace face à l'Allemagne en cas de guerre ${ }^{11}$. Au printemps 1935, le général Gamelin affirmait ainsi, dans une note destinée au ministre de la Guerre : « en ce qui concerne une guerre contre l'Allemagne, la Pologne est le seul pays qui, sur terre, peut être d'une aide vraiment efficace immédiatement $»^{12}$. Dans cette même note, le général Gamelin estimait que le soutien soviétique ne pourrait être que limité - actions aériennes au-dessus de l'est du territoire allemand et ravitaillement de la Pologne. En raison du poids stratégique accordé au facteur polonais en cas de conflit avec l'Allemagne, le général Gamelin et l'EMA pesèrent de tout leur poids en vue de favoriser les accords de Rambouillet de septembre 1936, par lesquels la France s'engageait à financer la modernisation des forces armées polonaises ${ }^{13}$.

\section{MARS 1938-SEPTEMBRE 1938 : L'ALLIANCE FRANCO-POLONAISE À L'ÉPREUVE DE LA CRISE DES SUDĖTES, OU L'IMPOSSIBLE TRIANGLE PARIS-VARSOVIE-PRAGUE}

Dès les lendemains de l'Anschluss, le général Gamelin et le $2^{\mathrm{e}}$ bureau de l'EMA ${ }^{14}$ relevèrent les possibilités stratégiques accrues de l'Allemagne face à la Tchécoslovaquie $^{15}$. Confrontés à l'hypothèse d'un conflit européen débutant par une offensive allemande en Tchécoslovaquie, les chefs militaires français considéraient dans leur très grande majorité que le sort de la Tchécoslovaquie dans les premières semaines d'un conflit avec l'Allemagne résulterait de la position que prendrait la Pologne. En

«Renseignement $-\mathrm{A} / \mathrm{s}$. des relations entre la Pologne et la Tchécoslovaquie », Section des renseignements (ci-après : SR) de l'EMA, 2 octobre 1936, « Renseignement-A/s. de la politique étrangère de la Pologne », SR de l'EMA, 6 octobre 1936, " Renseignement - A/S de la Politique étrangère de la Pologne », SR de l'EMA, 11 juin 1937 ; SHD-AT, 7N 3106, « Compte-Rendu de renseignements apportés par le Cdt de Villelume », sans indication d'origine, 17 février 1937.

11 G.-H. Soutou, «L'alliance franco-polonaise (1925-1933) ou comment s'en débarrasser ? », Revue d'histoire diplomatique, 1981/2-3-4, p. 295-348.

12 SHD-M, 1BB 8/38, lettre du général Gamelin au ministre de la Guerre, 6 avril 1935.

13 N. Jordan, op. cit., p. 170-174.

${ }^{14} \mathrm{Au}$ sein de chaque état-major général, le $2^{\mathrm{e}}$ bureau était en charge de l'analyse du renseignement et des relations avec les armées étrangères.

15 SHD-AT, 5N 579, « Note au sujet de la réalisation de l'Anschluss », général Gamelin, 14 mars 1938, et « Note sur la situation militaire actuelle dans le monde ", général Gamelin, 29 mars 1938 ; SHD-AT, 7N 2522, «La Tchécoslovaquie devant le nouvel empire allemand », $2^{\mathrm{e}}$ bureau de l'EMA, sans date mais probablement rédigé durant la seconde quinzaine de mars 1938, et « Note sur l'intervention allemande en Autriche », 2 bureau de l'EMA, 17 mars 1938. 
mars 1938, le général Mittelhauser, chargé au Conseil supérieur de la Guerre (CSG) ${ }^{16}$ de suivre les questions d'Europe centrale, concluait sa critique d'un exercice sur cartes par cette formule lapidaire : « Seule la Pologne peut apporter à la Tchécoslovaquie une aide efficace. [...] Une Pologne hostile, ce serait l'étranglement immédiat ; une Pologne neutre, ce serait l'asphyxie à brève échéance $»^{17}$. Cette opinion était également celle du $3^{\mathrm{e}}$ bureau de l'EMA et des généraux Armengaud, de l'Armée de l'Air, et Gamelin ${ }^{18}$. Ce dernier l'avait, d'ailleurs, rappelé au président du Conseil Édouard Daladier dans une note datée du 14 mars 1938.

Toutefois, aux lendemains de l'Anschluss, la Pologne ne semblait pas du tout disposée à s'engager dans la voie d'un soutien militaire au voisin tchécoslovaque. Cette réticence de Varsovie envers une entente polono-tchécoslovaque s'expliquait, en partie, par la persistance de la querelle des minorités entre Prague et Varsovie ${ }^{19}$. Bien plus, l'origine même de cette querelle semblait de nature à placer la Pologne, au moins sur les plans diplomatique et politique, dans le camp allemand. Aussi, dès le 21 mars, le lieutenant-colonel Gauché, chef du $2^{\mathrm{e}}$ bureau de l'EMA et, par ailleurs, très polonophile, fit part à l'attaché militaire britannique de sa vive préoccupation quant à la position adoptée par la Pologne, laquelle « se montrait définitivement germanophile $»^{20}$. Dans une note rédigée le lendemain, le chef du $2^{\mathrm{e}}$ bureau de l'EMA soulignait avec inquiétude que la Pologne semblait se placer dans la suite de l'Allemagne en vue de gagner des bénéfices immédiats, sans égard pour les intérêts de son alliée française et au risque de devenir complice d'un dépècement de la Tchécoslovaquie $^{21}$. Le lieutenant-colonel Gauché tirait les conséquences de cette attitude en envisageant une révision des accords de Rambouillet. À la fin du mois de mars 1938, la Pologne ravivait la querelle avec Prague au sujet des agissements des communistes tchécoslovaques, polonais et ukrainiens, puis, à partir de la mi-mai, les tensions au sujet des minorités de Tchécoslovaquie, ce qui renforça les craintes de l'EMA ${ }^{22}$.

La crise germano-tchécoslovaque des 20-21 mai aggrava le pessimisme du $2^{\mathrm{e}}$ bureau de l'EMA, qui releva que la Pologne avait pris des mesures militaires sur ses frontières avec l'URSS et la Tchécoslovaquie ${ }^{23}$. En conséquence, l'éventualité d'une

16 Le Conseil supérieur de la Guerre, présidé par le ministre de la Guerre, comprenait le commandant en chef des forces terrestres désigné, ainsi que les commandants de groupes d'armées et d'armées désignés.

17 SHD-AT, 1NN 83, « Conclusions du Général Mittelhausser [sic], Commandant du Parti Vert (Petite Entente, Pologne), à la séance finale de l'exercice du CSG 1937-1938 ».

18 I. Davion, op. cit., p. 398-399 et 403 ; SHD-AT, 5N 579, « Note au sujet de la réalisation de l’Anschluss », général Gamelin, 14 mars 1938.

19 I. Davion, op. cit., p. 416-419.

20 Public Record Office, Foreign Office (ci-après : PRO, FO), 371/21713, lettre de Victor Perowne à William Strang, 21 mars 1938.

${ }_{21}$ SHD-AT, 7N 3012, « Note sur l'attitude polonaise en face de l'Anschluss », $2^{\mathrm{e}}$ bureau de l'EMA, 22 mars 1938.

22 I. Davion, op. cit., p. 416-417.

23 G. Castellan, «L'alerte du 21 mai 1938, vue par le 2e bureau de l'EM français », Études européennes. Mélanges offerts à Victor-Lucien Tapié, Paris, Publications de la Sorbonne, "Études », 1973, p. 565 ; SHD-AT, 7N 2522, « Note pour le commandement », 2 bureau de l'EMA, 20 mai 1938. 
complicité germano-polonaise en vue de conduire une agression contre la Tchécoslovaquie gagnait en crédibilité. Un renseignement apporté par la SR-SCR laissait prévoir une occupation polonaise immédiate de Teschen en cas d'attaque allemande en Tchécoslovaquie $^{24}$. Il fallut attendre le mois de juillet 1938 pour obtenir quelques apaisements sur l'orientation du haut commandement et du gouvernement polonais ${ }^{25}$.

Dans les derniers jours du mois d'août 1938, la crise des Sudètes entra dans sa phase aigüe, et les EMG reçurent de nombreux renseignements concordants portant sur l'imminence d'une action de force allemande contre la Tchécoslovaquie ${ }^{26}$. Ces renseignements étaient de natures diverses : politique, militaire, économique. Les renseignements militaires indiquaient que, bien loin de se livrer à de traditionnelles manœuvres d'été, les forces militaires allemandes étaient en cours de mobilisation camouflée. Par ailleurs, la concentration de la Luftwaffe sur le pourtour de la Tchécoslovaquie était relevée. La date de l'agression projetée semblait fixée au 25 septembre 1938, ou à une date proche de ce jour. Les achats massifs de produits agricoles sur les marchés mondiaux confirmaient que le Reich se préparait à un conflit armé.

Dans ce contexte, les EMG parisiens s'interrogèrent sur la position qu'adopteraient les principales puissances européennes en cas de guerre, en particulier la Pologne. Celle-ci adopta une attitude très variable au cours du mois de septembre 1938, mais essentiellement inspirée par une interprétation littérale de son alliance avec $\mathrm{Pa}$ ris et par le souci de s'assurer des gains immédiats à Teschen ${ }^{27}$. Jusqu'à la mi-septembre, toutefois, le $2^{\mathrm{c}}$ bureau de l'EMA estimait que la Pologne gardait une attitude "réservée $»^{28}$. Par ailleurs, certaines déclarations antérieures de l'attaché militaire polonais à Paris permettaient d'espérer qu'en cas de conflit germano-tchécoslovaque, la Pologne prendrait le parti de la France ${ }^{29}$.

À partir du 21 septembre, et alors qu'une solution négociée au détriment de la Tchécoslovaquie semblait de plus en plus probable, la Pologne fit montre d'une agressivité marquée envers sa voisine méridionale. À cette date, Varsovie concentra des forces militaires à la frontière tchécoslovaque en face de Teschen et exigea for-

${ }^{24}$ SHD-AT, 7N 3107, « Pologne - Intentions au sujet de la Tchécoslovaquie », SR de 1'EMA, 23 mai 1938.

25 SHD-AT, 7N 2522, « Rapport sur la liaison de $2^{\mathrm{e}}$ bureau effectuée à Varsovie du 11 au 16 juillet $1938 », 2^{\mathrm{e}}$ bureau de l'EMA, 18 juillet 1938 ; SHD-AT, 7N 3015, « Compte-rendu », 2 bureau de l'EMA, sans date mais relatant une visite de l'attaché militaire polonais du 28 juillet 1938, et « Collaboration militaire franco-polonaise », $2^{\mathrm{e}}$ bureau de 1'EMA, juillet 1938 ; SHD-AT, 7N 3024, « Renseignement - A/s. Allemagne et Tchécoslovaquie », SR de l'EMA, 5 juillet 1938, et « Renseignement - A/s. de l'attitude de la Pologne et de la Yougoslavie », SR de l'EMA, sans date mais probablement rédigé aux alentours du 15 septembre 1938 .

26 SHD-AT, 7N 2523, dossier « Échéance Septembre (I) - 23.8. au 7.9.38 » et « Bulletin de renseignements - Allemagne ", $2^{\mathrm{e}}$ bureau de l'EMA, 30 août 1938 ; Documents diplomatiques français, $2^{\mathrm{e}}$ série, tome 10, Paris, Imprimerie Nationale, 1976, documents n 458 et n 506, 25 et 30 août 1938, p. 819-821 et 885-889.

27 I. Davion, op. cit., p. 426-440 ; H. Rollet, op. cit., p. 301 et suivantes.

28 SHD-AT, 7N 3015, «Attitude de la Pologne et de la Roumanie à l'égard du problème tchéco-slovaque [sic] $», 2^{\mathrm{e}}$ bureau de l'EMA, 13 septembre 1938.

${ }_{29}$ SHD-AT, 7N 3015, « Compte-rendu », $2^{\mathrm{e}}$ bureau de l'EMA, sans date mais probablement rédigé le 28 ou le 29 juillet 1938 . 
mellement la cession des territoires tchécoslovaques à population majoritairement polonophone ${ }^{30}$. Ces initiatives étaient accompagnées d'une démarche de l'attaché militaire polonais à Paris, qui déclara au lieutenant-colonel Gauché que « la question tchécoslovaque [était] à la veille d'être réglée », et qu'à l'issue de ce règlement « il restera[it] peut-être une Bohême neutre, mais [que] ce n' [était] pas sûr $\aleph^{31}$. Le lendemain, la Pologne interrompit les communications entre son territoire et la Tchécoslovaquie - imitant ainsi l'Allemagne -, ce qui provoqua une réaction de l'URSS, qui avertit le 23 septembre Varsovie qu'une agression contre la Tchécoslovaquie entraînerait ipso facto la dénonciation de l'accord polono-soviétique de non-agression de $1932^{32}$.

À Paris, ces mesures polonaises furent perçues comme des indices de la complicité de Varsovie et de Berlin. Du glissement d'unités allemandes, initialement positionnées face à la Pologne, vers le sud, René Massigli, directeur des Affaires politiques au ministère des Affaires étrangères, déduisit que la Pologne, tablant sur l'abstention française, avait entrepris de s'associer à l'Allemagne dans le dépècement de la Tchécoslovaquie ${ }^{33}$. La restriction unilatérale de la coopération franco-polonaise en matière de renseignement contre l'Allemagne, annoncée à l'attaché militaire français le 24 septembre, ne pouvait que renforcer cette impression ${ }^{34}$.

Un apaisement allait survenir dans les derniers jours de septembre, suite aux pressions exercées par la diplomatie française sur Prague en vue d'obtenir la cession à la Pologne de la Silésie de Teschen ${ }^{35}$. Une fois les négociations polono-tchécoslovaques initiées à ce sujet, Varsovie prit l'engagement de ne pas franchir la frontière sans l'accord de Prague, ce qui écartait, en cas de conflit, l'hypothèse d'une hostilité polonaise. Cependant, rien ne laissait présager que la Pologne se joindrait en pareille occurrence à la France, et les $2^{\text {es }}$ bureaux de la Marine et de l'Armée penchaient pour la neutralité de Varsovie dans un conflit éventuel ${ }^{36}$. Or le secours polonais avait toujours été jugé déterminant pour la Tchécoslovaquie en cas d'agression allemande, et pour la France en cas de conflit franco-allemand, comme le président Daladier le rappela à ses interlocuteurs britanniques le 26 septembre ${ }^{37}$.

Jusqu'au terme de la crise, le général Gamelin souhaita préserver la possibilité d'un secours polonais à la Tchécoslovaquie. S'entretenant le 28 septembre en milieu

30 I. Davion, op. cit., p. 428 ; H. Rollet, op. cit., p. 305.

31 SHD-AT, 7N 3015, « Compte-rendu », lieutenant-colonel Gauché, 21 septembre 1938.

32 H. Rollet, op. cit., p. 305.

33 SHD-AT, 7N 3015, « Compte-rendu », 2 bureau de l'EMA, 23 septembre 1938.

34 Ministère des Affaires étrangères (ci-après : MAE), Papiers 1940, Cabinet Georges Bonnet, 12, «Attaché militaire de Varsovie », cabinet du ministre des Affaires étrangères, 24 septembre 1938.

35 I. Davion, op. cit., p. 429-432 ; P. Le Goyet, op. cit., p. 182 ; MAE, Papiers 1940, Cabinet Georges Bonnet, 12, note datée du 23 septembre, minuit vingt, portant un message téléphoné d’Émile Charvériat ; SHD-AT, 7N 3105, télégramme de Victor de Lacroix, lu par les généraux Gamelin et Georges, 25 septembre 1938.

36 SHD-M, 1BB 3/31, « Note de renseignement - Les impressions de Londres sur la situation politique (25-9-38) », 2 $2^{\mathrm{e}}$ bureau de l'EMGM, 26 septembre 1938 ; SHD-AT, 7NN 15, rapport de l'EMA, 27 septembre 1938.

37 PRO, FO, 371/21744, « Second meeting - 26th September - 11.20 a.m. ». 
d'après-midi avec l'attaché militaire soviétique, il formula, à l'attention du gouvernement soviétique, la « demande de ne rien faire contre la Pologne », visiblement en vue de préserver les chances d'un ralliement polonais ${ }^{38}$. L'alliance polonaise primait, dans l'esprit du chef d'EMG de la Défense Nationale, sur un possible appui soviétique. Cet espoir du chef de l'EMG de l'Armée d'une intervention militaire polonaise en faveur de la Tchécoslovaquie peut sembler, au vu du comportement diplomatique de Varsovie en 1938, tout à fait irréaliste. Pourtant, les travaux d'Anna Cienciala démontrent que les dirigeants polonais, et en particulier le colonel Beck, n'écartaient pas l'éventualité d'effectuer une volte-face complète en cas d'attaque allemande contre la Tchécoslovaquie, pourvu que la France tînt ses engagements envers Prague ${ }^{39}$.

$\mathrm{Au}$ sommet de la crise, alors qu'une guerre paraissait imminente, le général Gamelin prit l'initiative d'écrire au maréchal Rydz-Smigły afin de lui rappeler les termes de leurs échanges verbaux de l'été 1936, dans le cadre de la négociation des accords de Rambouillet. À ce moment, le chef d'EMG de l'Armée avait reçu diverses assurances de son interlocuteur polonais : la Pologne n'attaquerait jamais la Tchécoslovaquie ; en cas d'agression allemande contre la Tchécoslovaquie, la Pologne demeurerait fidèle aux engagements prévus dans le pacte de la SDN et à la convention militaire franco-polonaise de 1921, laquelle prévoyait une concertation des états-majors en cas de «menaces de guerre » dont l'Allemagne serait l'origine ${ }^{40}$. Une source interne à la délégation polonaise accrédite la version du général Gamelin et contredit celle de l'ambassadeur de Pologne à Paris Juliusz Łukasiewicz, proche du colonel Beck, selon laquelle aucune contrepartie politique - au moins en ce qui concernait la Tchécoslovaquie - n'aurait été exigée en échange de l'engagement financier français ${ }^{41}$. Or, dans sa réponse datée du 3 octobre, le maréchal Rydz-Smigly affirma que « dans ces négociations [de l'été 1936], la question tchécoslovaque n'[avait] pas été posée comme condition, ni ajoutée d'aucune autre manière $»^{42}$. Aux tentatives du général Gamelin de donner une consistance à l'alliance franco-polonaise à la faveur de la crise des Sudètes, le chef de l'armée polonaise opposait une fin de non-recevoir.

38 SHD-AT, 1K 224/26, agenda du général Gamelin, 28 septembre 1938.

39 A.M. Cienciala, «The Munich crisis of 1938 : plans and strategy in Warsaw in the context of the western appeasement of Germany », Diplomacy \& Statecraft, vol. 10, n 2-3, 1999, p. 59-65.

40 Centre d'accueil et de recherche des Archives nationales (ci-après : CARAN), 496 AP/7, lettre du général Gamelin au ministre de la Défense Nationale et de la Guerre, 2 octobre 1936 ; SHD-AT, $1 \mathrm{~K}$ 224/26, agenda du général Gamelin, 6 septembre 1936 ; MAE, Papiers 1940, Cabinet Georges Bonnet, 10, lettre du général Gamelin au ministre de la Défense Nationale et de la Guerre, 2 octobre 1936.

41 N. Jordan, op. cit., p. 174.

42 SHD-AT, 5N 579, lettre du maréchal Rydz-Smigly au général Gamelin, 3 octobre 1938. 


\section{OCTOBRE 1938-MARS 1939 : LA RÉAPPRÉCIATION DU FACTEUR MILITAIRE ET POLITIQUE POLONAIS À L'AUNE DE LA RÉÉVALUATION DU POTENTIEL SOVIÉTIQUE, UN DÉBAT MAJEUR AU SEIN DES HAUTES INSTANCES MILITAIRES FRANÇAISES}

Au lendemain de la signature des accords de Munich, le général Gamelin tira le bilan de la situation politique et stratégique résultant de ces accords. Au cœur de la crise, le chef d'EMG de la Défense Nationale avait valorisé le potentiel militaire de la Tchécoslovaquie auprès des dirigeants français et britanniques ${ }^{43}$. Le $1^{\text {er }}$ octobre 1938, il estimait que la Tchécoslovaquie ne représentait plus, « militairement, qu'un facteur tout-à-fait de second plan $»^{44}$. En conséquence, la destruction finale de la Tchécoslovaquie en mars 1939 ne remit pas en cause les conceptions du chef d'EMG de la Défense Nationale, qui tenait déjà comme un fait acquis la disparition de la Tchécoslovaquie de l'échiquier militaire européen ${ }^{45}$. Cette disparition, combinée au développement de la ligne Siegfried, conduisait le général Gamelin à adopter une approche beaucoup plus prudente de l'offensive projetée sur le sol allemand ${ }^{46}$.

L'éclipse de la Tchécoslovaquie n'entraîna pas un regain de faveur pour la Pologne. Au contraire, au lendemain des accords de Munich, le général Gamelin se montra très amer et fustigea la "duplicité " polonaise ${ }^{47}$. Soupçonneux, il s'interrogeait : « la Pologne, dont la duplicité s'est manifestée dans la crise récente, [...] n'a-t-elle pas déjà accepté de se maintenir dans le sillage de l'Allemagne moyennant de larges compensations en Lithuanie ou en Ukraine ? $»^{48}$. Tout en reconnaissant que « l'importance de la Pologne, au point de vue militaire, rest[ait] pour [la France]

43 Documents diplomatiques français, 1932-1939, seconde série, tome 11, 3 septembre-2 octobre 1938, Paris, Imprimerie Nationale, 1977, document n 405, 27 septembre 1938, p. 612-613 ; SHD-AT, 2N 227, « Conversation avec Monsieur Neville Chamberlain du 26 septembre 1938 », général Gamelin, sans date ; SHD-AT, 7N 2522, CARAN, 496 AP/10, « Note au sujet du mémorandum allemand présenté à M. Chamberlain le 23 septembre $1938 », 2^{\text {e }}$ bureau de l'EMA, 25 septembre 1938 ; CARAN, 496 AP/8, « Munich (Suite) - par Édouard Daladier », p. 7-8; PRO, Cabinet Office, 23/95, « Cabinet 44 (38) - Conclusions of a Meeting of the Cabinet [...] on Monday 26th September, 1938, at 12 Noon », note annexe « Statement made by the Prime Minister to the Cabinet on Monday, 26th September, (Cabinet 45 (38)) as to information conveyed to him that morning by general Gamelin ».

44 SHD-AT, 1NN 10, « Le bilan (notes du général Gamelin après les accords de Munich) », $1^{\text {er }}$ octobre 1938 .

45 SHD-AT, 1 N 38, « Procès-verbal de la séance d'études [du CSG] du 13 mars 1939 », sans indication d'origine.

46 S. Catros, « La stratégie générale et opérationnelle du général Gamelin en 1938 : nouvelles sources, nouvelle approche », Stratégique, n 110, décembre 2015, p. 31-44 ; SHD-AT, 7N 2506, « Bulletin de renseignements - Allemagne - L'armée allemande au $1^{\text {er }}$ janvier $1939 », 2^{\mathrm{e}}$ bureau de l'EMA, janvier-février 1939 ; SHD-AT, 7N 2688, " Note sur les fortifications allemandes (avec croquis) », $2^{\text {e }}$ bureau de l'EMA, 31 mai 1939 ; SHD-AT, 7N 3720, «Directive pour le général commandant le théâtre d'opérations du Nord-Est en vue des opérations initiales à conduire éventuellement entre Rhin et Moselle », général Gamelin, 31 mai 1939.

47 SHD-AT, 1NN 10, "Le bilan (notes du général Gamelin après les accords de Munich) », $1^{\text {er }}$ octobre 1938 .

48 SHD-AT, 5N 579, « Note sur la situation actuelle », général Gamelin, 12 octobre 1938. 
considérable », et sans envisager la dénonciation pure et simple de l'alliance qui liait Paris et Varsovie, il proposa de prendre quelques distances avec la Pologne, notamment en suspendant l'application des accords de Rambouillet ${ }^{49}$. Le général Bührer, chef d'EMG des Colonies, émit également le vœu que les capitaux dispensés à la Pologne dans le cadre des accords de 1936 furent attribués à la création d'une industrie de guerre dans les colonies françaises ${ }^{50}$.

L'alliance polonaise comptait cependant des partisans enthousiastes au sein du haut commandement français. Le général Vuillemin, chef d'EMG de l'Armée de l'Air, préconisa une " rupture radicale avec les Soviets » de nature, selon lui, à rapprocher la Pologne et l'Italie de la France ${ }^{51}$. Le général Colson, second adjoint du général Gamelin, prit, sur un plan plus large, position en faveur du maintien des relations étroites avec les États de l'Est européen - dont il excluait l'URSS, taxée d' « incapacité militaire » et soupçonnée d'entretenir des projets subversifs pour l'ordre européen ${ }^{52}$. Ainsi, le général Colson refusait d' " abandonner délibérément la carte polonaise ». Passées les frictions de la crise des Sudètes, la Pologne lui semblait au contraire à même de constituer le pivot d'un groupement d'États comprenant les pays baltes et l'Entente balkanique capable de s'opposer au Drang nach Osten. L'alliance polonaise paraissait donc toujours, pour l'EMAA comme pour le second adjoint du général Gamelin, la pierre d'angle de la politique française en Europe centrale et orientale.

L'amiral Darlan, chef d'EMG de la Marine, ne partageait pas ces vues. Tout en reconnaissant que " les desseins de l'Allemagne [étaient] certainement l'expansion vers l'Est, le Sud-Est et la Méditerranée orientale », il ne faisait aucune mention de l'alliance polonaise ou du rôle de la Pologne dans une coalition opposée à l'Allemagne ${ }^{53}$. Ce silence est plus significatif dès lors que l'on a connaissance d'une note rédigée en janvier suivant par l'amiral ${ }^{54}$. Intitulée « L'Angleterre et la France peuvent-elles soutenir un conflit contre l'Allemagne et l'Italie ? ", cette note préconisait une politique de « repli impérial » sur l'empire colonial français, au détriment de la défense des positions et des alliances françaises en Europe orientale. Il préconisait ainsi, implicitement, un abandon des engagements pris par Paris envers Varsovie. Cependant, la portée de cette note est difficile à évaluer. D'une part, l'on ne connait pas l'ampleur de sa diffusion. D'autre part, l'amiral Darlan ne prit plus, par la suite, de position de cette nature.

Quoiqu'il en soit, ces divergences ne devaient pas avoir d'impact sur l'orientation de la politique étrangère et stratégique de la France. Les renseignements dont disposaient les états-majors français durant l'automne et l'hiver qui suivirent Munich indi-

49 SHD-AT, 5N 579, lettre du général Gamelin à Édouard Daladier, 12 octobre 1938.

50 SHD-AT, 5N 579, lettre du général Bührer au général Gamelin, 19 octobre 1938.

51 SHD-AT, 5N 579, lettre du général Vuillemin au général Gamelin, 25 octobre 1938.

52 SHD-AT, 5N 579, « Note sur la situation actuelle », général Colson, sans date mais probablement rédigée peu avant le 26 octobre 1938.

53 SHD-AT, 5N 579, lettre de l'amiral Darlan au général Gamelin, 17 octobre 1938.

54 SHD-M, 1BB 2/208, «L'Angleterre et la France peuvent-elles soutenir un conflit contre l'Allemagne et l'Italie? », amiral Darlan, 22 janvier 1939. 
quaient une prochaine poussée à l'Est de l'Allemagne au détriment de la Pologne ${ }^{55}$. Certains signes laissaient espérer aux états-majors français un rapprochement polono-soviétique dans un contexte de menace allemande croissante ${ }^{56}$. La conclusion d'un accord commercial entre Varsovie et Moscou au mois de février 1939 fut interprétée comme l'étape préparatoire à une collaboration industrielle et commerciale en cas de guerre - fourniture de chars, d'avions et de munitions soviétiques à l'armée polonaise ${ }^{57}$.

Dès lors, la Pologne redevint, aux yeux de l'EMA, un partenaire essentiel dans le système de sécurité français, au titre d'allié de revers. Confrontée aux ambitions italiennes et allemandes, la France devait pouvoir compter sur le soutien militaire polonais au début d'un conflit, afin d'éviter de devoir supporter initialement tout le poids de la pression militaire allemande et italienne. Dès les premiers jours de janvier 1939, les généraux Gamelin et Dentz informèrent le Quai d'Orsay, en la personne du directeur des Affaires politiques et commerciales, Émile Charvériat, qu'ils « estim[aient] comme primordiale la valeur de l'appui polonais $»^{58}$. La neutralité de la Pologne, entraînant celle de la Roumanie, permettrait à l'Allemagne de disposer d'une carte de guerre initiale extrêmement favorable et de consacrer la totalité de ses forces militaires à une offensive contre la France. Une note du $2^{\mathrm{e}}$ bureau de l'EMA, rédigée au mois de janvier 1939, corroborait ce point de vue, et rappelait les éléments de la puissance militaire terrestre polonaise ${ }^{59}$.

L'EMAA semble avoir également manifesté, au début de l'année 1939, un regain d'intérêt pour l'alliance polonaise. Une mission de l'EMAA se rendit en Pologne au mois de février 1939 et conclut que « la Pologne, sans avoir une puissance aérienne comparable à celles des grandes Puissances Européennes, constitu[ait] cependant dans l'Europe Orientale un élément de résistance à l'expansion allemande, dans la

55 P. Paillole (colonel), Services spéciaux, 1935-1945, Paris, Robert Laffont, 1975, p. 117 ; SHDAT, 7N 2515, « Note sur la déclaration commune polono-soviétique », extraite du rapport du ministre de la semaine du 21 au 27 novembre 1938, 2 ${ }^{\mathrm{e}}$ bureau de l'EMA; SHD-AT, 1N 43, lettre du général Gamelin au président du Conseil, 19 décembre 1938, et note annexe « Synthèse des propos tenus par une haute personnalité militaire allemande $», 2^{\mathrm{e}}$ bureau de l'EMA, sans date.

56 SHD-AT, 7N 2515, « Note sur la déclaration commune polono-soviétique », extraite du rapport du ministre de la semaine du 21 au 27 novembre 1938, 2 bureau de l'EMA, et « Source : Attaché Militaire à Moscou - URSS - Amélioration des relations polono-soviétiques », rapport du ministre de la semaine du 16 au 22 janvier 1939, 2 bureau de l'EMA ; SHD-AT, 7N 2522, « Considérations sur la constitution d'un bloc oriental », lieutenant-colonel Gauché, 22 décembre 1938.

57 G. Vidal, Ennemi intérieur, enjeu stratégique et politique de sécurité : l'armée française et le problème bolchevik de la révolution russe au régime de Vichy (octobre 1917-novembre 1942), vol. III du dossier préparé sous la direction d'Olivier Forcade et Jean-François Muracciole et présenté en vue de l'obtention d'une HDR en Histoire contemporaine en l'Université de Montpellier-III Paul-Valéry, automne 2011, p. 433 ; P. de Villelume (général, auteur) et S. Catros (éd.), De Munich à Dantzig, Journal (30 août 1938-18 août 1939), Paris, Presses de l’Université Paris-Sorbonne, « Mondes contemporains », 2015, p. 155.

58 SHD-AT, 7N 3015, « Note », 2 bureau de l'EMA, 5 janvier 1939.

59 Documents diplomatiques français, $2^{\mathrm{e}}$ série, tome 13 (ci-après DDF-II-13), Paris, Imprimerie Nationale, 1979, document n 462, 30 janvier 1939, p. 832-833 ; SHD-AT, 7N 3130, " Pologne », $2^{\mathrm{e}}$ bureau de l'EMA, sans date. 
mesure du moins où les Puissances Occidentales lui viendr[aie]nt en aide $\nu^{60}$. Cette appréciation particulièrement élogieuse fut reprise dans les semaines qui suivirent par les généraux Vuillemin et Mendigal, ainsi que par le lieutenant-colonel de Vitrolles ${ }^{61}$. L'EMAA rejoignait donc l'EMA dans la défense de l'alliance polonaise. Ce point de vue influença jusqu'au cabinet du ministre de la Défense Nationale et de la Guerre ${ }^{62}$.

Ce regain d'intérêt pour la Pologne, son armée et son apport potentiel au sein d'une coalition opposée à l'Allemagne était partagé par tous les officiers de l'EMA et de l'EMAA. Cependant, ces officiers ne partageaient par la même conception de l'intérêt que présentait la Pologne. Pour les généraux Vuillemin et Colson, la Pologne représentait à l'Est la seule puissance sur laquelle la France pouvait et devait s'appuyer, tandis que l'URSS était vouée aux gémonies ${ }^{63}$. Le général Georges partageait ces vues et affirmait à l'été 1939 que la France et la Pologne, alliées à la Roumanie, n'avaient pas besoin de l'URSS pour s'opposer à une agression allemande ${ }^{64}$. Pour ces officiers, l'alliance polonaise constituait avant tout le moyen d'écarter l'alliance soviétique, rejetée essentiellement par anticommunisme.

Ces conceptions, faisant de la Pologne l'atout-maître de la France et l'étai majeur d'un front oriental en devenir, constituaient la doxa au sein de l'EMA depuis le début des années trente. Elles furent cependant progressivement remises en question à partir de la fin de l'année 1938 par plusieurs hauts responsables de l'EMA, sur la base d'une réappréciation du facteur militaire soviétique ${ }^{65}$. Ainsi, à l'automne 1938, le $2^{\mathrm{e}}$ bureau relevait les indices d'une dépolitisation et d'une stabilisation du haut commandement et de l'outil militaire soviétiques, durement ébranlés par deux années de purges ${ }^{66}$. Sur ces bases, le $2^{\mathrm{e}}$ bureau de l'EMA, et en particulier le commandant Rudloff, en charge des questions soviétiques à partir de l'été 1938, eut tendance à ré-

60 SHD-AT, 2N 235, rapport de la mission de l'EMAA en Pologne effectuée du 7 au 18 février 1939, « Conclusions générales ».

${ }_{61}$ SHD-AT, 2N 235, lettre du général Vuillemin au général Gamelin, 20 mars 1939, et bordereau d'envoi au ministère de la Défense Nationale et de la Guerre d'un rapport de l'attaché de l'Air en Pologne, annotation du général Mendigal, 17 avril 1939 ; SHD-AT, 7N 3180, « Conférence sur l'aviation de l'URSS et les puissances secondaires de l'Europe centrale », lieutenant-colonel de Vitrolles, février 1939.

62 SHD-AT, 5N 579, « Note sur l'aviation polonaise », section de Défense Nationale du cabinet militaire du ministre de la Défense Nationale et de la Guerre, 5 mai 1939 ; SHD, Armée de l'Air (ci-après : SHD-AA), 2B 106, « Note établie par l'État-major de l'Armée de l'Air », sans date, mais probablement rédigée aux alentours du 10 avril 1939.

63 SHD-AT, 5N 579, « Note sur la situation actuelle », général Colson, sans date mais probablement rédigée peu avant le 26 octobre 1938, et lettre du général Vuillemin au général Gamelin, 25 octobre 1938.

64 P. de Villelume (général, auteur) et S. Catros (éd.), op. cit., p. 377.

65 Sur cette question des relations entre l'armée française et l'URSS : G. Vidal, Une alliance improbable. L'armée française et la Russie soviétique, 1917-1939, Rennes, PUR, « Histoire politique de la France au XX ${ }^{\mathrm{e}}$ siècle », 2015, 307 p.

${ }_{66}$ G. Vidal, Ennemi intérieur, enjeu stratégique et politique de sécurité..., op. cit., p. 161 ; SHDAT, 7N 2515, « Source : Presse - URSS - Renforcement de l'autorité du personnel de commandement dans l'armée soviétique », extrait du rapport du ministre pour la semaine du 10 au 16 octobre 1938, $2^{\text {e }}$ bureau de l'EMA, et « Source : Attaché Militaire - URSS - Promotions dans le Haut Commandement de l'armée soviétique », extrait du rapport du ministre pour la semaine du 27 février au 5 mars 1939 , $2^{\text {e }}$ bureau de l'EMA. 
évaluer à la hausse la qualité de l'outil militaire soviétique, couramment sous-estimé jusqu'à l'été $1938^{67}$. Le lieutenant-colonel Gauché partageait les vues de son subordonné, et louait à partir de décembre 1938 la puissance et les capacités de manœuvre de l'armée soviétique ${ }^{68}$. Sur le plan politique et diplomatique, il semblait de plus en plus probable que l'URSS pencherait en faveur des puissances occidentales en cas de conflit européen. Les purges ayant touché l'Armée rouge et les milieux politiques et administratifs de Moscou furent interprétées à partir de 1938 dans divers milieux diplomatiques et militaires à Paris comme résultant de la volonté de Staline d'éradiquer toute tendance germanophile au sein des milieux dirigeants ${ }^{69}$.

Une tendance opposée s'exprimait toutefois au sein des milieux du haut commandement et des EMG. La Section de renseignements, et notamment l'officier chargé des questions russes, le commandant Josset, ainsi que le sous-chef de l'EMA en charge des relations avec les armées étrangères, le général Dentz, persistaient à considérer avec scepticisme le développement du potentiel militaire soviétique ${ }^{70}$. Enfin, divers renseignements alimentaient le soupçon de duplicité à l'endroit du Kremlin, accusé alternativement ou simultanément de fomenter un accord secret avec Berlin et de souhaiter imposer le bolchevisme à une Europe épuisée par une longue guerre ${ }^{71}$.

${ }^{67}$ G. Vidal, Une alliance improbable..., op. cit., 307 p.; SHD-AT, 7N 3148, « Visite du chef du SR polonais à Paris en Décembre 1938 (Compte-rendu du SR français communiqué sous n 1007 du 3.1.39) ", sans auteur, et « Renseignement - URSS - a.s/ de la valeur de la RKKA », SR de l'EMA, 13 février 1939 ; SHD-AT, 7N 3186, « Coopération éventuelle de l'URSS », commandant Rudloff, 22 juillet 1939.

68 SHD-AT, 7N 2522, " Tableau récapitulatif des forces armées de la Pologne, de la Roumanie et de l'URSS », annexe aux « Considérations sur la constitution d'un bloc oriental », lieutenant-colonel Gauché, 22 décembre 1938 ; SHD-AT, 7N 3434, « URSS - Composition de l'armée », commandant Rudloff, sans date, probablement rédigé à la fin de l'année 1938.

69 SHD-AT, 7N 3124, lettre de l'ambassadeur de France à Moscou au ministre des Affaires étrangères, 15 décembre 1937, et « Mémento pour le Colonel Gauché », anonyme et sans date, mais sans doute rédigé par le commandant Krebs dans la seconde quinzaine de décembre 1937 ; SHD-AT, 1NN 10, «Exposé fait le 30 mai 1938 par le capitaine de vaisseau R. de Villaine devant les Officiers de Réserve du $2^{\mathrm{e}}$ bureau de l'État-Major Général », p. 8-11; SHD-M, 1BB 2/175, «Bulletin du haut commandement n 31 », SE de l'EMGM, 18 janvier 1938; PRO, FO, 371/20702, rapport de Fitzroy Maclean, 18 décembre 1937.

70 R. Porte (lieutenant-colonel), « Renseignement militaire et relations internationales : le rôle des attachés militaires et la prise de décision politico-militaire, illustrés par l'exemple des négociations franco-soviétiques de 1938-1939», F. Guelton (colonel, dir.) et A. Bicer (lieutenant, dir.), Naissance et évolution du renseignement dans l'espace européen (1870-1940). Entre démocratie et totalitarisme, quatorze études de cas, Paris, Service historique de la Défense, 2006, p. 376-378 ; SHD-AT, 7N 3146, dépêches du colonel Palasse au ministre de la Défense Nationale et de la Guerre, 18 février et 12 juillet 1938 ; SHD-AT, 7N 3148, « Renseignement - A/S. Puissance militaire de la Russie », SCM, 3 novembre 1938 ; SHD-AT, 7N 3186, « Observations suggérées par le rapport du colonel Palasse traitant de la situation intérieure de l'URSS en 1937 », 2e bureau de l'EMA, sans date ; SHD-AT, 4K 4, témoignage du colonel Krebs, plages 6-10.

${ }_{71}$ SHD-AT, 7N 2523, " Compte-rendu des renseignements recueillis par le SR le 20 octobre 1938 », $2^{\mathrm{e}}$ bureau de 1'EMA; SHD-AT, 7N 3127, « Compte-rendu des renseignements recueillis par la SR le 30 Novembre $1938 », 2^{\mathrm{e}}$ bureau de l'EMA; SHD-AT 1N 47, « Compte-rendu de renseignements », lieutenant-colonel Gauché, 22 décembre 1938, p. 2 ; SHD-AT, 7N 2515, « Source : Attaché Militaire URSS - Accords économiques entre l'URSS d'une part, l'Allemagne et l'Italie d'autre part », extrait du rapport du ministre de la semaine du 27 février au 5 mars 1939, $2^{\mathrm{e}}$ bureau de l'EMA; SHD-AT, 7N 
À la fin du mois de juillet 1939, le général Georges, premier adjoint du général Gamelin, affirmait en privé que la Russie soviétique était une « planche pourrie $»^{72}$.

Cependant, si le point de vue favorable à une alliance avec Moscou n'était pas partagé par tous au sein de l'EMA, il détermina nettement les conceptions du général Gamelin $^{73}$. À l'instar du lieutenant-colonel Gauché, le chef d'EMG de la Défense Nationale estimait que la Pologne ne pourrait soutenir la lutte contre l'Allemagne dans la durée qu'à la condition d'être ravitaillée par l'URSS ${ }^{74}$. Ainsi, par un renversement de perspective total, le général Gamelin, qui, au milieu des années trente et jusqu'à la crise des Sudètes, faisait du rapprochement avec Moscou un complément utile mais non impératif de l'alliance franco-polonaise, conditionnait au premier semestre 1939 l'utilité de l'alliance polonaise au soutien soviétique ${ }^{75}$.

Sur le plan technique, l'étude détaillée établie au début de juin 1939 par le général Billotte, membre du CSG, pointait clairement les faiblesses militaires de la Roumanie et de la Pologne et soulignait «l'insuffisance évidente des forces polonaises et roumaines livrées à leur propre sort » face à l'Allemagne ${ }^{76}$. En conséquence, en cas d'offensive germano-hongroise contre la Roumanie et la Pologne, ces dernières semblaient hors d'état de résister durablement sans l'appoint « d'un nombre important - 30 à 35 - de grandes unités; de tous les éléments blindés et de réserve générale qui leur [faisaient] défaut ; d'une aviation nombreuse ; du matériel de remplacement ; des matières premières » qui ne pouvaient être fournies que par l'URSS.

Finalement, l'historien doit constater que dès le printemps 1939, la Pologne ne constituait plus le point d'ancrage essentiel, l'allié principal, dans la stratégie française en Europe centrale et orientale. Pour certains, tenants d'une ligne antisoviétique, l'alliance polonaise ne présentait qu'un intérêt d'opportunité - elle permettait de justifier l'inutilité de l'alliance soviétique. Pour d'autres, dont le général Gamelin, chef d'état-major général de la Défense Nationale, la Pologne se présentait désormais comme un allié, certes nécessaire, mais fragile et incapable de se défendre durablement face à l'Allemagne en dehors d'un soutien soviétique.

2522, "Considérations sur la constitution d'un bloc oriental », lieutenant-colonel Gauché, 28 décembre 1938 ; Documents on British Foreign Policy, 1919-1939, $3^{\mathrm{e}}$ série, vol. IV, Londres, H. M. Stationery Office, 1951, document n 555, p. 535 ; SHD-AT, 7N 3024, « Renseignement - Source : Z », SR de l'EMA, 11 mai 1939 ; SHD-AT, 7N 3122, « Renseignement - URSS - Au sujet des déclarations de l'ex-officier soviétique Krivitzky », SR de l'EMA, 25 mai 1939, p. 3-8 et 34-36; SHD-AT, 7N 2571, « Compte-rendu de renseignements militaires du 22 Juillet $1939 », 2^{\mathrm{e}}$ bureau de l'EMA.

72 P. de Villelume (général, auteur) et S. Catros (éd.), op. cit., p. 376.

73 R. Porte, op. cit., p. 376-378.

74 DDF-II-13, op. cit., documents n 178 et n 462, 19 décembre 1938 et 30 janvier 1939, p. 338 et 832-833 ; SHD-AT, 7N 2522, « Considérations sur la constitution d'un bloc oriental », lieutenant-colonel Gauché, 28 décembre 1938 ; SHD-AT, 7N 3130, « Pologne », $2^{\mathrm{e}}$ bureau de l'EMA, sans date.

75 SHD-AT, 1K 224/8, note manuscrite du général Gamelin datée du 20 décembre 1933 et «Position générale de 1'Armée française », janvier 1934 ; SHD-AT, 7N 3558, "Positions générales de l'Armée française », note manuscrite dictée par le général Gamelin, juin 1934 ; CARAN, 351 AP/3, « Réunion du Conseil supérieur de la guerre -9 mars $1936 »$.

76 SHD-AT, 1N 43, lettre du général Billotte au chef d'état-major général de l'Armée, 3 juin 1939, et rapport annexé « Note au sujet d'actions militaires éventuelles en Europe centrale ». 


\section{AVRIL 1939-AOÛT 1939 : DEVANT LE DANGER ALLEMAND, LA RÉSURRECTION DE L'ALLIANCE ?}

Si le haut commandement français relativisait nettement la puissance militaire polonaise, l'intérêt de l'alliance polonaise demeurait cependant essentiel pour la France. Dans l'hypothèse d'un conflit européen résultant d'une offensive initiale allemande à l'ouest, le soutien polonais permettait d'écarter l'hypothèse d'un écrasement initial et rapide des armées françaises par la Wehrmacht. Une partie significative des forces allemandes serait divertie du front ouest pour défendre l'est du territoire allemand ou attaquer la Pologne. En outre, l'hypothèse d'une entente germano-italo combinée à une entente avec Varsovie ou à une neutralité polonaise dès l'ouverture d'un conflit représentait un véritable cauchemar stratégique pour l'EMA ${ }^{77}$. Ce raisonnement explique la démarche du général Gamelin qui s'adressa le 29 avril 1939 au maréchal Rydz-Smigly - soit le lendemain de la dénonciation par Hitler de la déclaration de non-agression de janvier 1934 - pour proposer la tenue de conversations militaires secrètes bilatérales ${ }^{78}$.

Nous ne reviendrons pas sur la teneur générale et les dispositions particulières de ces conversations qui se déroulèrent au milieu du mois de mai 1939. Dans le cadre de notre étude, un constat s'impose : le but du haut commandement français était d'arrimer Varsovie à Paris, alors que des rumeurs persistantes prêtaient au colonel Beck des projets de rapprochement avec Berlin ${ }^{79}$. Ainsi, le général Vuillemin affirma à ses interlocuteurs polonais que « l'aviation française [pouvait] au début d'un conflit agir vigoureusement pour dégager la Pologne », alors qu'il était pleinement conscient des graves faiblesses de l'Armée de l'Air. Les généraux Gamelin et Georges firent cependant montre de davantage de réserve : ils promirent au ministre des Affaires militaires polonais un franchissement rapide de la frontière allemande, mais refusèrent d'envisager une attaque contre les fortifications du Westwall avant la troisième semaine de mobilisation ${ }^{80}$. Un extrait des souvenirs du colonel Paillole semble confirmer définitivement que ces conversations constituaient un outil pour le haut commandement français en vue d'ancrer définitivement la Pologne dans le camp opposé à l'Allemagne. Si l'on en croit les souvenirs de celui qui était alors adjoint au chef du contre-espionnage militaire français, le gouvernement français ordonna que les agents doubles infiltrés au sein du dispositif allemand de renseignement diffusassent

77 SHD-AT, 2N 20, «Étude sur l'établissement de plans de Défense Nationale dans les différentes hypothèses de conflit », comprenant deux documents annexes, $2^{\mathrm{e}}$ et $3^{\mathrm{e}}$ bureaux de l'EMA, 22 juin 1936.

78 M.S. Alexander, op. cit., p. 305.

79 R. Meltz, Alexis Léger dit Saint-John Perse, Paris, Flammarion, « Grandes biographies », 2008, p. 549 ; P. de Villelume (général, auteur) et S. Catros (éd.), op. cit., p. 266 ; SHD-AT, 7N 2516, « Bulletin de renseignements $n 10 », 2^{\mathrm{e}}$ bureau de l'EMA, 10 mai 1939.

80 M.S. Alexander, op. cit., p. 305-312 ; M. Gamelin (général), Servir, tome II, « Le prologue du drame, 1930-août 1939 », Paris, Plon, 1946, p. 413-424 Totally suppress ; SHD-AT, 2N 235, procès-verbaux des conversations d'état-major franco-polonaises, mai 1939 ; SHD-AT, 5N 579, « Protocole résumant les conversations tenues les 15, 16 et 17 Mai 1939 [...] », généraux Gamelin et Kasprzycki, 19 mai 1939. 
l'information de la venue en France du ministre polonais des Affaires militaires, le général Kasprzycki ${ }^{81}$.

Dès lors, ne faut-il voir dans l'attitude du haut commandement français que cynisme machiavélique, visant à compromettre la Pologne afin de mieux l'abandonner à son sort par la suite ? Il est difficile de répondre de façon tranchée à cette question. Certes, plusieurs responsables militaires français n'hésitaient pas à envisager, au printemps et à l'été 1939, un abandon pur et simple de la Pologne - et de l'URSS - aux ambitions allemandes. Le maréchal Pétain, le général Georges, premier adjoint du général Gamelin, les généraux Condé et Prételat, membres du CSG, et le général Mendras, commandant de l'École supérieure de Guerre, partageaient tous cette opinion $^{82}$. L'instruction personnelle et secrète émise par le général Georges, commandant désigné pour le front Nord-Est, en juillet 1939 prévoyait explicitement une « attaque à objectif rapproché sur une avancée de la position fortifiée » au trentième jour suivant la mobilisation, allongeant ainsi considérablement les délais de réalisation des promesses faites à la délégation polonaise en mai $1939^{83}$.

Cependant, les convictions de ces hauts responsables militaires n'entravèrent en rien la détermination du général Gamelin, lequel entreprit, au printemps et à l'été 1939, de gagner le soutien soviétique à la cause franco-anglo-polonaise. Dans l'esprit du chef d'état-major général de la Défense Nationale, un affrontement franco-allemand était, de toute façon, inéluctable à brève échéance ${ }^{84}$. Dans cette optique, la France avait tout intérêt à ce que la guerre commençât à l'est de l'Europe - afin d'éviter un écrasement initial rapide de l'armée française - et qu'elle y durât le plus longtemps - afin de développer au maximum le potentiel militaire et industriel franco-britannique. Il était ainsi essentiel pour le général Gamelin que la Pologne disposât d' " une armée forte ${ }^{85}$ et fût en mesure de tenir le plus longtemps possible face à l'Allemagne, avec le soutien soviétique. Pour cette raison, le général Gamelin insista pour que les demandes de crédits ou d'armements de la Pologne fussent satisfaites en priorité par rapport à celles des autres alliés de la France ${ }^{86}$. Toutefois, conscient des limites de la production française d'armement et des besoins de l'armée française, le général Gamelin souhaitait favoriser l'acquisition de matériel soviétique par les Polonais ${ }^{87}$.

${ }_{81}$ P. Paillole (colonel), op. cit., p. 117.

82 J.-L. Crémieux-Brilhac, Les Français de l'An 40, tome I, op. cit., p. 35 et 115, et tome II, « Ouvriers et soldats », Paris, Gallimard, « La Suite des temps », 1990, p. 377 ; P. de Villelume (général), op. cit., p. 8 et 16 ; P. de Villelume (général, auteur) et S. Catros (éd.), op. cit., p. 310 et 359.

83 SHD-AT, $1 \mathrm{~N} 46$ et $7 \mathrm{~N} 3715$, « Instruction personnelle et secrète pour le commandant du groupe d'armées en vue des opérations initiales à conduire éventuellement entre Rhin et Moselle », général Georges, 24 juillet 1939 .

84 J.-L. Crémieux-Brilhac, Les Français de l'An 40, tome I, p. 31.

85 SHD-M, 1BB 2/183, « Procès-verbal des conversations franco-britanniques du 13 Juillet 1939 ».

86 SHD-AT, 2N 225, « Procès-verbal de la Réunion des chefs d'états-majors généraux du Vendredi 16 Juin 1939 »; SHD-AT, 5N 579, lettres du général Gamelin au président du Conseil, 23 juin et 11 août 1939.

87 SHD-AT, 2N 225, « Procès-verbal de la Réunion des chefs d'états-majors généraux du Vendredi 16 Juin 1939 », « Procès-verbal de la Réunion des chefs d'états-majors généraux du 11 Juillet 1939 » 
Ainsi, tout au long du printemps et de l'été 1939, le général Gamelin œuvra en faveur d'un rapprochement entre Moscou et les alliés franco-britanniques, conçu comme préparatoire à un rapprochement polono-soviétique ${ }^{88}$. Le général Gamelin joua un rôle essentiel dans la reprise du contact avec Moscou au printemps 1939 : non seulement il ordonna au général Palasse, attaché militaire à Moscou, de sonder l'état-major soviétique en vue de connaître les possibilités de ravitaillement de la Pologne et de la Roumanie en matériel de guerre, mais il se rendit personnellement à l'ambassade d'URSS pour convaincre ses interlocuteurs qu'il n'était pas trop tard pour mettre un coup d'arrêt aux ambitions hitlériennes ${ }^{89}$. Durant l'été 1939 , il envisagea avec son homologue britannique les modalités de l'intervention militaire soviétique dans le camp allié lors du conflit à venir ${ }^{90}$. Il fit part, en privé, de son irritation devant les tergiversations des diplomaties britanniques et françaises et émit le souhait d'une conclusion rapide et positive des négociations anglo-franco-soviétiques, y compris au prix de l'acceptation de la plupart des exigences de Moscou'

Cependant, les espoirs et tentatives du général Gamelin ne rencontrèrent que peu de succès. D'une part, les Soviétiques, par la voix du maréchal Vorochilov et du commissaire aux Affaires étrangères Litvinov, refusèrent dès le printemps 1939 d'envisager la fourniture de matériel militaire à l'armée polonaise ${ }^{92}$. D'autre part, les Polonais, approchés à plusieurs reprises par les Français, refusèrent toujours de prendre contact avec les Soviétiques, sur le plan de la fourniture d'armement ou sur le plan opérationnel ${ }^{93}$. En conséquence, le général Gamelin n'envisagea à aucun moment de contraindre les alliés polonais à prendre langue avec Moscou. À la mi-juillet 1939, devant ses subordonnés, il précisa qu' « il faudra[it] traiter ces problèmes [de coopération polono-soviétique] avec précaution car les Polonais n'accepter[aient] pas le

et lettre du général Gamelin au président du Conseil, 15 mai 1939 ; SHD-AT, 2N 236, lettre du général Gamelin au président du Conseil, 15 avril 1939 ; SHD-M, 1BB 2/183, « Procès-verbal des conversations franco-britanniques du 13 Juillet $1939 »$.

88 SHD-AT, 1N 44, « Compte-rendu de la Réunion des Chefs d’État-Major [des généraux membres du CSG] le 19 Avril à 15 heures ».

${ }^{89}$ M.J. Carley, « End of the "Low, Dishonest Decade" : Failure of the Anglo-French-Soviet Alliance in 1939 », Europe-Asia Studies, vol. 45, n 2, 1993, p. 319 ; Documents diplomatiques français, 1932-1939, seconde série, tome 15, 16 mars-30 avril 1939, Paris, Imprimerie Nationale, 1981, document n 344, 11 avril 1939, p. 550 ; SHD-AT, 7N 3186, télégramme du général Gamelin au général Palasse, 11 avril 1939.

90 SHD-M, 1BB 2/183, « Procès-verbal des conversations franco-britanniques du 13 Juillet 1939 ».

${ }_{91}$ P. de Villelume (général, auteur) et S. Catros (éd.), op. cit., p. 360, 369 et 374.

92 SHD-AT, 7N 3186, télégramme du général Gamelin au général Palasse, 11 avril 1939, « Note au sujet de contacts éventuels entre États-Majors français et soviétique », note manuscrite probablement rédigée par le commandant Rudloff, sans date, et « Note au sujet de contacts éventuels entre États-Majors français et soviétique ", note dactylographiée, $2^{\mathrm{e}}$ bureau de l'EMA, sans date.

93 J.-B. Duroselle, Politique étrangère de la France : La Décadence, 1932-1939, Paris, Imprimerie Nationale, "Points Histoire », 1979, p. 473-474; Documents diplomatiques français, 2 ${ }^{\mathrm{e}}$ série, tome 18, Paris, Imprimerie Nationale, 1985, documents n 147, n 150, n 169 et n 284, 19, 20 et 23 août 1939, p. 189, 191, 214-215 et 343 ; SHD-AT, 2N 235, rapport de la mission de l'EMAA en Pologne effectuée du 7 au 18 février 1939, "Échange de renseignements sur l'aviation allemande »; SHD-AT, 7N 3186, télégramme du général Gamelin à l'attaché militaire de France en Pologne, 19 août 1939, et lettre du général Dentz au général Palasse, 21 août 1939 ; SHD-AA, 8 Z 20, témoignage du général Bailly, bande 1, face 2. 
principe d'occupation par les troupes russes de leur territoire $»^{94}$. Le chef d'EMG de l'Armée ajoutait cependant immédiatement qu' " en cas de guerre ils ser[aient] bien obligés de l'accepter ». Ainsi, probablement sous l'influence des rapports envoyés par l'ambassade de France à Varsovie, le général Gamelin était persuadé qu'une fois le conflit déclenché, les Polonais accepteraient le soutien militaire, au moins sur le plan aérien, que ne manquerait pas d'offrir $\mathrm{Moscou}^{95}$.

Devant la volte-face soviétique que laissait présager l'annonce le 22 août de l'envoi d'une délégation allemande à Moscou, le général Gamelin maintint son point de vue sur l'utilité du maintien des engagements français envers la Pologne. D'une part, l'offensive militaire allemande en Pologne permettrait à l'armée française de mobiliser sans craindre d'attaque surprise dans les premières semaines du conflit. Une fois la Pologne vaincue par l'Allemagne, le risque d'une offensive éclair allemande contre la France n'en serait qu'accentué, l'Allemagne bénéficiant alors des ressources polonaises et, vraisemblablement, du pétrole roumain ${ }^{96}$. D'autre part, sur le plan diplomatique, le général Gamelin soulignait la détermination de Londres et le risque, si la France faisait preuve de faiblesse et n'assumait pas ses engagements, d'un retournement d'alliance débouchant sur une entente germano-britannique ${ }^{97}$. Enfin, le général Gamelin mit en avant le principe de la parole donnée, affirmant que « la France se déshonorerait si elle ne tenait pas ses engagements envers la Pologne $\rangle^{98}$. Le général Colson, second adjoint du général Gamelin, résumait ainsi la situation le $1^{\text {er }}$ septembre 1939 : « nous sommes dans l'engrenage politique ; d'ailleurs, si nous laissions aujourd'hui écraser la Pologne, nous nous retrouverions dans quelques semaines devant une situation encore plus grave et encore plus seuls $»^{99}$.

94 SHD-AT, 2N 225, « Procès-Verbal de la réunion des Chefs d'État-Major du 17 Juillet 1939 ».

95 Documents diplomatiques français, $2^{\mathrm{e}}$ série, tome 17, Paris, Imprimerie Nationale, 1984, documents n 240 et n 403, 19 juillet et 2 août 1939, p. 409-411 et 672-677 ; SHD-AT, 1K 608, « Souvenirs militaires » du général Olivier Poydenot, tome III, « L'Entre-deux-guerres », p. 227-228; SHD-AT, 1N 44, « Compte-rendu de la réunion des Chefs d'État-Major [du CSG] le 19 Avril à 15 Heures »; SHD-AT, 7N 3186, lettre du général Gamelin au général Doumenc, 27 juillet 1939 ; MAE, Papiers 1940, Papiers Rochat, 31, télégrammes de Léon Noël datés des 13 avril et 14 mai 1939.

96 CARAN, 496 AP/44, déposition du général Gamelin, 28 septembre 1940, et « Conditions dans lesquelles a été déclarée et s'est développée la guerre de 1939 », général Gamelin, sans date mais probablement rédigé à l'automne 1940 ; CARAN, 496 AP/45, « Mémoire 6 - Pièce 2 (Suite) - Dépositions de M. Georges Bonnet (dossier 10) (Déposition du 29 Avril) », général Gamelin, 8 mai 1941.

97 É. du Réau, Édouard Daladier, 1884-1970, Paris, Fayard, «Pour une histoire du XX' siècle », 1993, p. 360 ; CARAN, 496 AP/44, « Conditions dans lesquelles a été déclarée et s'est développée la guerre de 1939 », général Gamelin, sans date mais probablement rédigé à l'automne 1940.

98 CARAN, 496 AP/30, «Interrogatoire du général Gamelin », note manuscrite d’Édouard Daladier, sans date.

99 M.-H. Gauché (général), Le Deuxième bureau au travail (1935-1940), Paris, Amiot-Dumont, 1953, p. 104. 


\section{CONCLUSION}

Considérée jusqu'au printemps 1938 comme l'allié continental principal de la France en cas de conflit, la Pologne ne put conserver ce statut jusqu'à l'aube de la guerre. Si les évolutions de la diplomatie du colonel Beck suscitèrent incompréhension et irritation au sein des EMG parisiens, elles ne motivèrent pas un abandon des engagements contractés envers Varsovie, en dépit des velléités de certains hauts responsables militaires. Le facteur militaire polonais demeurait après la conférence de Munich essentiel dans la stratégie militaire française - en particulier au sein de l'EMA -, mais il fut davantage conçu dans le cadre plus large d'un vaste front oriental où l'URSS jouerait progressivement le rôle prépondérant. La disparition du facteur tchécoslovaque à Munich et l'accroissement subséquent de la domination allemande en Europe centrale transformaient radicalement la situation stratégique. Devant l'écroulement du rêve lancinant depuis les années 1920 d'un triangle stratégique Paris-Varsovie-Prague, le général Gamelin favorisa la constitution d'un triangle Paris-Moscou-Varsovie. Le refus de Varsovie d'entrer dans ces vues et, surtout, la brusque volte-face soviétique du 23 août 1939, condamnaient le schéma élaboré par le général Gamelin. L'offensive en Sarre, conçue dès le printemps 1939 comme une simple opération de diversion visant à retenir à l'ouest le nombre le plus élevé possible de divisions allemandes, ne fut qu'un geste sans lendemain destiné à sauver l'honneur.

\section{SOURCES ET BIBLIOGRAPHIE}

\section{Ouvrages}

Alexander M.S., The Republic in danger. General Maurice Gamelin and the politics of French defence, 1933-1940, Cambridge, Cambridge UP, 1992, 573 p.

Carré C. (général), Histoire du ministère de la Défense, Panazol, Lavauzelle, 2001, 582 p.

Chaix B. (général), En mai 1940, fallait-il entrer en Belgique ? Décisions stratégiques et plans opérationnels de la campagne de France, Paris, Economica, " Campagnes et stratégies. Les grandes batailles », 2000, XII-349 p.

Crémieux-Brilhac J.-L., Les Français de l'An 40, tome I, « La guerre, oui ou non ? » et tome II, « Ouvriers et soldats », Paris, Gallimard, « La Suite des temps », 1990, 647 p. et $740 \mathrm{p}$.

Davion I., Mon voisin, cet ennemi. La politique de sécurité française face aux relations polono-tchécoslovaques entre 1919 et 1939, Bruxelles/Berne/Berlin, PIE Peter Lang, «Enjeux internationaux », 2009, 472 p.

Du Réau É., Édouard Daladier, 1884-1970, Paris, Fayard, " Pour une histoire du XX siècle », 1993 
Dessberg F., Le triangle impossible, les relations franco-soviétiques et le facteur polonais dans les questions de sécurité en Europe (1924-1935), Bruxelles, PIE Peter Lang, « Enjeux internationaux », 2009, 440 p.

Duros elle J.-B., Politique étrangère de la France : La Décadence, 1932-1939, Paris, Imprimerie Nationale, « Points Histoire », 1979, 568 p.

Dutailly H. (colonel), Les problèmes de l'armée de terre française (1935-1939), Paris, Imprimerie Nationale, 1980, 449 p.

Jordan N., The Popular Front and Central Europe. The dilemmas of French impotence, 1918-1940, Cambridge, Cambridge UP, XV-348 p.

Le Goyet P. (colonel), Le mystère Gamelin, Paris, Presses de la Cité, 1975, 379 p.

May E.R., Strange Victory. Hitler's conquest of France, New York, Hill and Wang, 2000, VIII-594 p.

Meltz R., Alexis Léger dit Saint-John Perse, Paris, Flammarion, « Grandes biographies », 2008, 846 p.

Merglen A. (général), Les forces allemandes sur le front de l'Ouest en septembre 1939, thèse rédigée sous la direction de Georges Dupeux et soutenue en l'Université de Bordeaux, 1969, $161 \mathrm{p}$.

Rollet H., La Pologne au XX siècle, Paris, A. Pedone, 1985, 603 p.

Vidal G., Ennemi intérieur, enjeu stratégique et politique de sécurité : l'armée française et le problème bolchevik de la révolution russe au régime de Vichy (octobre 1917-novembre 1942), vol. III du dossier préparé sous la direction d'Olivier Forcade et Jean-François Muracciole et présenté en vue de l'obtention d'une HDR en Histoire contemporaine en l’Université de Montpellier-III Paul-Valéry, automne 2011, 689 p.

Vidal G., Une alliance improbable. L'armée française et la Russie soviétique, 1917-1939, Rennes, PUR, « Histoire politique de la France au XXe siècle », 2015, 307 p.

\section{Articles et contributions à des ouvrages collectifs}

Carley M.J., " End of the "Low, Dishonest Decade" : Failure of the Anglo-French-Soviet Alliance in $1939 »$, Europe-Asia Studies, vol. 45, n 2, 1993, 303-341 p.

Castellan G., "L'alerte du 21 mai 1938, vue par le 2e bureau de l'état-major français », Études européennes. Mélanges offerts à Victor-Lucien Tapié, Paris, Publications de la Sorbonne, « Études », 1973, p. 556-566.

Catros S., « La stratégie générale et opérationnelle du général Gamelin en 1938 : nouvelles sources, nouvelle approche », Stratégique, n 110, décembre 2015, p. 31-44.

Cienciala A.M., " The Munich crisis of 1938 : plans and strategy in Warsaw in the context of the western appeasement of Germany », Diplomacy \& Statecraft, vol. 10, n 2-3, 1999, p. $48-81$.

Porte R. (lieutenant-colonel), «Renseignement militaire et relations internationales : le rôle des attachés militaires et la prise de décision politico-militaire, illustrés par l'exemple des négociations franco-soviétiques de 1938-1939 », Guelton F. (colonel, dir.) et Bicer A. (lieutenant, dir.), Naissance et évolution du renseignement dans l'espace européen (1870-1940). Entre démocratie et totalitarisme, quatorze études de cas, Paris, Service historique de la Défense, 2006, p. 365-388.

Soutou G.-H., « L'alliance franco-polonaise (1925-1933) ou comment s'en débarrasser ? », Revue d'histoire diplomatique, 1981/2-3-4, p. 295-348. 


\section{Mémoires, souvenirs et sources imprimées}

De Villelume P. (général), Journal d'une défaite, 23 août 1939-16 juin 1940, Paris, Fayard, «Les grandes études contemporaines », 1976, XXVI-478 p.

De Villelume P. (général, auteur) et Catros S. (éd.), De Munich à Dantzig, Journal (30 août 1938-18 août 1939), Paris, Presses de l'Université Paris-Sorbonne, « Mondes contemporains $», 2015,453 \mathrm{p}$.

Documents diplomatiques français, seconde série, Paris, Imprimerie Nationale, 19 tomes, 1963-1986.

Documents on British Foreign Policy, 1919-1939, deuxième série, Londres, H. M. Stationery Office, volumes XII-XIX, 1972-1982.

Gamelin M. (général), Servir, tome II, « Le prologue du drame, 1930-août 1939 », Paris, Plon, 1946

Gauché M.-H. (général), Le Deuxième bureau au travail (1935-1940), Paris, Amiot-Dumont, 1953, $239 \mathrm{p}$.

Noë1 L., Polonia Restituta, la Pologne entre deux mondes, Paris, Publications de la Sorbonne/Institut d'Études Slaves, 1984, XVI-288 p.

Paillole P. (colonel), Services spéciaux, 1935-1945, Paris, Robert Laffont, 1975, 565 p. 